\title{
The Concept of Majorization in Experimental Designs
}

\author{
Miltiadis S. Chalikias \\ Department of Business Administration, Piraeus University of Applied Science, Greece \\ Email: mchalikias@hotmail.com, mchalik@teipir.gr
}

\begin{abstract}
The information of interest is contained in the variance matrix, $\mathbf{V}=\sigma^{2} \mathbf{Q}^{-1}$, or equivalently in the information matrix $\mathbf{Q}$. The problem of finding estimators minimizing a decreasing convex functional of the variance matrix or maximizing an increasing concave functional of the information matrix is crucial in statistics. There are several optimality criteria in bibliography. We explain how the concept of Majorization can be used to investigate optimal experimental designs.
\end{abstract}

Keywords: Majorization, direct effects, carry-over effects, universally optimality, F-Optimality, repeated measurements designs, saturated designs, row-column, factorial designs

\section{Introduction-Existing Knowledge}

The experimental designs are used to estimate the parameters of interest in a stochastic model. A classic book on this subject is the book by W.G. Cochran and G.M. Cox (1957).

The research of optimal designs actually started by the 1950. In 1958 Kiefer extended Wald's research on D-optimization of Latin square designs in a wider context which uses the Generalized Youden Designs (GYD).

The concept of universally optimal designs was given by Kiefer (1975 a), who generalized the idea of a Balanced Block Design (BBD) and showed when the GYD is universally optimal. Also he worked on their construction (Kiefer, 1975 b) and generalized the results of the work of Agrawal (1966), Hartley and Smith (1948), Shrikhande (1951).

A lot of paper followed, giving optimal design for the estimation of the parameters or optimal designs for the comparison of treatments with one or more controls.

An overview of results in homogeneous and heterogeneous populations given by Hedayat, Jacroux and Majundar (1988), and some treatments compared with a control examined by Bechhofer and Tamhane (1981). There followed a lot of work in this area, such as those of Hedayat and Majumbar (1985), Jacroux (1987), Stufken (1987), etc.

Optimal designs with dependent observations were examined by Martin and Eccleston (1993), Morgan and Uddin (2003), etc.

Bibliography on finding and constructing universally optimal designs and optimal designs satisfying some criteria are quite extensive. Interesting is the case of two treatments with independent observations and with even number of units (Kounias and Chalikias 2008a, 2015; Chalikias and Kounias 2012 , 2017). In contrast, the bibliography on cases of three or more treatments is limited (Chalikias 2017). This happens because when the number of treatments is $v \geq 3$, the theoretical and computational aspects demand a different approach.

In this paper the row designs with homogeneous populations under dependence will be investigated especially the Balance Block Designs and the row-column designs with two and three treatments and depended observations are mentioned. Moreover we will examine the comparisons of $v$ treatments with a standard treatment under dependence.

Kiefer (1975 a) introduced a new method to generalize the existing results. An improved approach on the same subject is given in the book by F. Pukelsheim (1993).

Many researchers worked with problems of optimal designs using the model with dependent observations, such as Chauhan (2000), Chauhan and Martin (2001), Kunert (1988), Martin and Eccleston (1993), Morgan and Uddin (1991), Uddin (1997), Uddin and Morgan (1991). 
Optimal row-column designs when the treatments are two with positive dependence between the observations have been examined by Morgan and Uddin (2002).

Pericleous and Kounias (2010) worked in finding universally optimal designs comparing v treatments with a standard (control) treatment. We examined cases where experimental units are homogeneous or heterogeneous with one characteristic. The concept of mazorization was applied and the universally optimal design was presented when the population is homogeneous. In heterogeneous populations Pericleous and Kounias first applied the concept of mazorization and then found A-, D- and MV-optimal designs.

The definitions of information function and information matrix were given in (Pericleous and Kounias, 2011), in the line followed by Pukelsheim (1993). The definitions of information function and information matrix are given. Homogeneous populations set in a row with dependent observations are examined and properties of the optimal designs are presented, two treatments are studied in more detail and the optimal designs are given. Non homogeneous populations by one characteristic set in p rows and q columns, with independent observations, are analyzed and examples of Balanced Treatments Block designs are given.

Row designs are examined (Pericleous and Kounias 2012) with three treatments, homogeneous population with dependent observations. The dependence follows a first order autoregression $(\mathrm{AR}(1))$. A filtering procedure was presented to reduce the number of competing designs.

In the case of optimal designs in $3^{k}$ fractional factorial for the estimating linear and quadratic contrasts, most of the works for constructing optimal designs for parameter estimation in fractional factorials are concentrated in factors at two levels. It is of interested estimating linear and quadratic contrasts, in fractional factorials, with each factor at three levels. The books by Dey and Mukerjee (1999) and $\mathrm{Wu}$ and Hamada (2000), cover the topic, with a lot references. If the number of runs is $\mathrm{N}=0 \bmod 9$, the orthogonal arrays, $\mathrm{OA}(\mathrm{N} ; \mathrm{k} ; 3 ; 2)$, are $\Phi$-optimal under different type of criteria, $\operatorname{Kiefer}(1958,1960)$. If $\mathrm{N}=1 \bmod 9$ the plan obtained by augmentation of a run to an $\mathrm{OA}(\mathrm{N}-1 ; \mathrm{k} ; 3 ; 2)$ is D-optimal, KolyvaMachera (1989a), G-optimal, Kolyva-Machera(1989b), and optimal under Cheng's type 1 criteria, Mukerjee (1999). These efforts were concentrated in adding runs to an $\mathrm{OA}(\mathrm{N} ; \mathrm{k} ; 3 ; 2)$ so that the resulting design is optimal in some sense.

Chatzopoulos et al. (2009), studied the optimality of designs obtained by adding $p$ runs to an orthogonal array for experiments involving $m$ factors each at $s$ levels. The optimality criterion used was the Type 1 criterion due to Cheng (1978) which is an extension of Kiefer (1975) universal optimality criterion. Unlike what happens with orthogonal array plus one run designs, the behavior of designs obtained via augmentation of an orthogonal array by $p$ runs depends on the particular runs added. Onea et al. (2009) investigated two techniques of constructing optimal weighing designs under different optimality criteria when the number of runs is $N \equiv 3(\bmod 4)$. The first method was presented by Chatzopoulos, Kolyva - Machera and Chaterjee (2009), who studied Type I optimality criteria for $\mathrm{s}^{\mathrm{m}}$ factorial designs obtained by adding p particular runs to an orthogonal array of strength two. In the present work the orthogonal arrays $\mathrm{OA}(\mathrm{N}-3, \mathrm{~m}, 2,2)$ are augmented by three runs. The second one is due to Ehlich (1964). In order to compare the two methods they constructed the corresponding information matrices, applied D-, E- and A- optimality criteria and explored their efficiency. Chatterjee et al. (2011) considered the issue of optimality of fractional factorial experiments involving $\mathrm{m}$ factors each at two levels. The optimality criteria used here are the type 1 criteria, which include the D- and Acriteria. It is shown that if there exists an orthogonal array $\mathrm{OA}(\mathrm{N}-\ell ; \mathrm{m} ; 2 ; 3), \ell=1,2$, then there exists an n-run type 1 optimal fractional factorial plan for a $2^{\mathrm{m}}$ experiment under a model that includes the mean, all main effects and all two-factor interactions with a factor in common. These plans are obtained by adding any one run to an $\mathrm{OA}(\mathrm{N}-1 ; \mathrm{m} ; 2 ; 3)$ for $N \equiv 3(\bmod 8)$ and two specific runs to an $\mathrm{OA}(\mathrm{N}-2 ; \mathrm{m} ; 2 ; 3)$ for $N \equiv 2(\bmod 8)$.

In this project we will deal with factorial designs with factors, each at 3 levels, the primary interest is the estimation of linear and quadratic contrasts of factor's effects. Orthogonal designs, called orthogonal balanced arrays (OBA), are derived for any value of the number of experimental runs, in which the estimators of linear contrasts are uncorrelated with those of quadratic contrasts.

Another issue of interesting in experimental designs is saturated designs. An experimental design is said to be saturated if all degrees of freedom are consumed by the estimation of parameters, leaving no degrees of freedom for error variance estimation. Saturated resolution III factorial designs are commonly used in screening experiments, to determine which of many factors affects the measure of pertinent 
quality characteristics. The purpose of this paper is to give saturated resolution III designs, minimizing the generalized variance of the main effects and the general mean, that is, D-optimal designs. In recent years, there has been a considerable interest in optimal saturated main effect designs. Mukerjee et al. (1986) and Kraft (1990) showed that in the two-factor case all such designs are equivalent with respect to D-optimality. Later Mukerjee and Sinha (1990) considered optimality results on almost saturated main effect designs in the two-factor case. The result was also obtained by Pesotan and Raktoe (1988) in the special case of $2^{m}$ factorials, who also worked on a subclass of $3^{m}$ factorials. The first attempt to extend the two factor results to three factors was done by Chatterjee and Mukerjee (1993). Later Chatterjee and Narasimhan (2002) using techniques from Graph Theory and Combinatorics, claimed about the upper bound of the determinant of the saturated $3 \times m_{2} \times m_{3}$ factorials. Chatzopoulos and Machera (2002) considered the class of $m_{1} \times m_{2} \times m_{3}, m_{1} \geq 2, m_{2} \geq m_{1}, m_{3} \geq m_{2}$ factorial plans with the minimum number of observations. Their interest was to find conditions in order to maximize the determinant of the information matrix and compare their findings with other researchers' findings. Chatzopoulos and Kolyva-Machera (2005), considered the problem of finding an algorithm in order to construct D-optimal saturated factorial designs with three factors. The interest was to estimate the general mean and main effects while all higher order interactions are negligible, that is Resolution III plans. In order to reduce the steps of the algorithm, they diminish the number of the cases and have to search by studying the design matrix. The algorithm they suggested, leads in finding the upper bound of the determinant of the design and moreover in constructing the D-optimal design. They apply an algorithm in order to find the D-optimal saturated $4 \times 4 \times m_{3}, m_{3} \geq 6$ design and compare their results with those are mentioned in bibliography. Moreover they verify the findings for the $4 \times 4 \times 4$ and $4 \times 4$ $\times 5$ D-optimal saturated designs and expand their results by applying the algorithm for the saturated 4 $\times 5 \times 5$ and $4 \times 5 \times 6$ designs. Moreover, Chatzopoulos and Kolyva-Macher (2006) extended the results concerning D-optimal saturated main effect designs for $2 \times m_{2} \times m_{3}$ to $3 \times m_{2} \times m_{3}$ factorials, when $3 \leq m_{2} \geq 6$ and $m_{3} \geq m_{2}$. Recently Karagiannis and Moyssiadis (2005, 2008) extended the above cases and studied the D-optimal $3 \times m_{2} \times m_{2}$ and $3 \times m_{2} \times\left(m_{2}+1\right)$ saturated designs.

Although the issue has been studied by many researchers it is not exhausted. This area of research is far from complete because of the difficulty of the calculations for designs that are not symmetrical. Optimal designs are found only in certain cases.

\section{Majorization}

The information of interest is contained in the variance matrix, $\mathbf{V}=\sigma^{2} \mathbf{Q}^{-1}$, or equivalently in the information matrix $\mathbf{Q}$. There are several optimality criteria in bibliography. The most frequently used optimality criteria are:

G-optimality: If $E\left(y_{i}\right)=f^{\prime}\left(\mathbf{x}_{i}\right) \theta, f\left(\mathbf{x}_{i}\right)=\left(f_{1}\left(\mathbf{x}_{i}\right), \ldots, f_{p}\left(\mathbf{x}_{i}\right)\right)^{\prime}, i=1, \ldots, n$ is the linear model, then the variance response surface in points $\mathbf{x}_{i}, i=1, \ldots, n$, is $\operatorname{var}\left(f^{\prime}\left(x_{i}\right) \hat{\theta}\right)=\sigma^{2} f^{\prime}\left(x_{i}\right) \mathbf{V} f\left(x_{i}\right)$ and for Goptimality: $\min _{d} \max _{i} f^{\prime}\left(\mathbf{x}_{i}\right) \mathbf{V} f\left(\mathbf{x}_{i}\right)$.

G-optimality criterion is "a prediction criterion". This criterion latterly is called global or Goptimality. The aim of G-optimality is a response estimation criterion and can be defined as minimizing the maximum variance of any predicted value over the experimental space.

\section{D-optimality: $\max _{d} \operatorname{det}\left(\mathbf{Q}_{d}\right)$.}

D-optimality is a quite popular criterion maximizing the determinant of the information matrix, in essence D-optimality chooses that design as the "best" for which the volume (expected volume) of the joint confidence ellipsoid of the parameters of interest is minimized.

A-optimality: $\min _{d}\left(\right.$ trace $\mathbf{V}_{d}$ ) or $\max _{d}\left(\text { trace } \mathrm{Q}_{d}^{-1}\right)^{-1}$.

A-optimality is defined as minimizing the average variance of the parameter estimates, that is, minimizing the average variance of the best linear unbiased estimator (BLUE) of the parameters of interest.

E-optimality: $\min _{d} \max _{i} \lambda_{i}(\mathbf{V})$ or $\underbrace{\max }_{d} \underbrace{\min }_{i} \lambda_{i}(\mathbf{Q})$. 
E-optimality is defined as maximizing the minimum eigenvalue of the information matrix or equivalently, minimizing the maximum eigenvalue of the inverse of the information matrix. The aim of E-optimality is to minimize the maximum variance of all possible normalized linear combinations of parameter estimates.

$\boldsymbol{M V}$-optimality: $\min _{d} \max _{i}\left(v_{i i}\right), \quad \mathbf{V}=\left(v_{i j}\right)$.

MV-optimality is defined as minimizing the maximum variance of the parameters of interest.

Instead of dealing with specific optimality criteria is preferable to work with universal optimality, an idea introduced by Kiefer (1975), and $\Phi$-optimality and if such designs do not exist, then go to the specific criteria mentioned above.

Universal and $\Phi$-optimality are defined below:

Definition 1: If $C \in \operatorname{nnd}(k)$ the function $\varphi(\mathbf{C})$ is called information function if $\varphi(\mathbf{C}) \rightarrow \mathbb{R}$, and the following conditions are satisfied:

(i) $\mathbf{C} \geq \mathbf{D} \Rightarrow \varphi(\mathbf{C}) \geq \varphi(\mathbf{D})$

(ii) $\varphi(p \mathbf{C}+(1-p) \mathbf{D}) \geq p \varphi(\mathbf{C})+(1-p) \varphi(\mathbf{D}) \quad \forall 0<p<1, \mathbf{C}, \mathbf{D} \geq \mathbf{0}$

(iii) $\varphi(d C)=d \varphi(C), \quad \forall d>0, \mathbf{C} \geq \mathbf{0} \Rightarrow \varphi(\mathbf{0})=0 \Rightarrow \varphi(\mathbf{C}) \geq 0, \forall \mathbf{C} \geq \mathbf{0}$

(iv) $\varphi(\mathbf{C})=\varphi\left(\mathbf{P C P}^{\prime}\right) \quad \forall \mathbf{C} \in \operatorname{nnd}(k), \mathbf{P} \in \operatorname{perm}(k)$

So the information functions are concave and increasing. Here $n n d(k)$ is for non negative definite $k \times k$ matrix, $\operatorname{perm}(k)$ is for permutation $k \times k$ matrix.

Definition 2: A design $d^{*}$ with information matrix $\mathbf{C}_{d}^{*}$ is universally optimal, in the class $\mathrm{F}$ of designs, if it maximizes $\varphi\left(\mathbf{C}_{d}\right), d \in F$, for all information functions $\varphi$.

Definition 3: A design $d^{*}$ with information matrix $\mathbf{C}_{d}^{*}$ is $\Phi$-optimal, in the class $\mathrm{F}$ of designs, if $\sum_{i=1}^{v} \varphi\left(\lambda_{i}\right) \leq \sum_{i=1}^{v} \varphi\left(\mu_{i}\right)$ for all continuous, decreasing convex functions $\varphi$, where $\lambda=\left(\lambda_{1}, \ldots, \lambda_{v}\right)$ are the latent roots of $\mathbf{C}_{d}^{*}$ and $\mu=\left(\mu_{1}, \ldots, \mu_{v}\right)$ are the latent roots of $C_{d} \forall d \in F$.

This is equivalent to: $\lambda \prec \mu$, that is, the vector $\lambda$ is majorized by the vector $\mu$ (Marshall and Olkin p.10).

If a design is universally optimal it is also $\Phi$-optimal and if a design is $\Phi$-optimal, it is A-, D-, Eoptimal. Also a design may be optimal for a criterion but not optimal for the other criteria. Note that MV-optimality is not covered by $\Phi$-optimality, since it cannot be expressed as a function of the latent roots of the information matrix.

The idea is to use the concept of majorization in order to establish $\Phi$ optimality.

\section{$3 \quad$ Fields of Applications}

They will be present in three fields of application of the concept of majorization, one for each different case of experimental designs.

1) The first idea is for Repeated measurements of p periods, two treatments, $2^{p}$ sequences (we use the symbolism $s_{i} i=0,1, \ldots, m$, where $\left.m=2^{p}-1\right)$.

For the estimation of the parameters we have:

If $\mathrm{n}$ is the total number of experimental units and $u_{i} i=0,1, \ldots, m$ is the number of experimental units that the sequence $s_{i}$ of treatments is applied, then $u_{0}+u_{1}+\ldots+u_{m}=n$. The model is:

$$
Y_{i j}=\tau_{d(i, j)}+\pi_{j}+\delta_{d(i, j-1)}+\gamma_{i}+e_{i j}
$$

where, $i=1, \ldots, n$, refers to the unit employed, $j=1, \ldots, p$ the period, $\tau_{d(i j)} \in\left\{\tau_{\mathrm{A}}, \tau_{\mathrm{B}}\right\}$ is the direct effect of the treatment applied, under design $\mathrm{d}$, in the $j$ th period on the ith unit, $\delta_{d(i, j-1)} \in\left\{\delta_{\mathrm{A}}, \delta_{\mathrm{B}}\right\}$ is the residual effect of the treatment applied the $(j-1)$ th period on the $i$ th unit, $\pi_{j}$ is the $j$ th period effect and $\gamma_{i}$ is the $i$ th unit effect. The errors $e_{i j}$ are independent within each unit and among units and have 0 mean and constant variance. 


\title{
The Concept of Majorization in Experimental Designs
}

\author{
Miltiadis S. Chalikias \\ Department of Business Administration, Piraeus University of Applied Science, Greece \\ Email: mchalikias@hotmail.com, mchalik@teipir.gr
}

\begin{abstract}
The information of interest is contained in the variance matrix, $\mathbf{V}=\sigma^{2} \mathbf{Q}^{-1}$, or equivalently in the information matrix $\mathbf{Q}$. The problem of finding estimators minimizing a decreasing convex functional of the variance matrix or maximizing an increasing concave functional of the information matrix is crucial in statistics. There are several optimality criteria in bibliography. We explain how the concept of Majorization can be used to investigate optimal experimental designs.
\end{abstract}

Keywords: Majorization, direct effects, carry-over effects, universally optimality, F-Optimality, repeated measurements designs, saturated designs, row-column, factorial designs

\section{Introduction-Existing Knowledge}

The experimental designs are used to estimate the parameters of interest in a stochastic model. A classic book on this subject is the book by W.G. Cochran and G.M. Cox (1957).

The research of optimal designs actually started by the 1950. In 1958 Kiefer extended Wald's research on D-optimization of Latin square designs in a wider context which uses the Generalized Youden Designs (GYD).

The concept of universally optimal designs was given by Kiefer (1975 a), who generalized the idea of a Balanced Block Design (BBD) and showed when the GYD is universally optimal. Also he worked on their construction (Kiefer, 1975 b) and generalized the results of the work of Agrawal (1966), Hartley and Smith (1948), Shrikhande (1951).

A lot of paper followed, giving optimal design for the estimation of the parameters or optimal designs for the comparison of treatments with one or more controls.

An overview of results in homogeneous and heterogeneous populations given by Hedayat, Jacroux and Majundar (1988), and some treatments compared with a control examined by Bechhofer and Tamhane (1981). There followed a lot of work in this area, such as those of Hedayat and Majumbar (1985), Jacroux (1987), Stufken (1987), etc.

Optimal designs with dependent observations were examined by Martin and Eccleston (1993), Morgan and Uddin (2003), etc.

Bibliography on finding and constructing universally optimal designs and optimal designs satisfying some criteria are quite extensive. Interesting is the case of two treatments with independent observations and with even number of units (Kounias and Chalikias 2008a, 2015; Chalikias and Kounias 2012 , 2017). In contrast, the bibliography on cases of three or more treatments is limited (Chalikias 2017). This happens because when the number of treatments is $v \geq 3$, the theoretical and computational aspects demand a different approach.

In this paper the row designs with homogeneous populations under dependence will be investigated especially the Balance Block Designs and the row-column designs with two and three treatments and depended observations are mentioned. Moreover we will examine the comparisons of $v$ treatments with a standard treatment under dependence.

Kiefer (1975 a) introduced a new method to generalize the existing results. An improved approach on the same subject is given in the book by F. Pukelsheim (1993).

Many researchers worked with problems of optimal designs using the model with dependent observations, such as Chauhan (2000), Chauhan and Martin (2001), Kunert (1988), Martin and Eccleston (1993), Morgan and Uddin (1991), Uddin (1997), Uddin and Morgan (1991). 
Optimal row-column designs when the treatments are two with positive dependence between the observations have been examined by Morgan and Uddin (2002).

Pericleous and Kounias (2010) worked in finding universally optimal designs comparing v treatments with a standard (control) treatment. We examined cases where experimental units are homogeneous or heterogeneous with one characteristic. The concept of mazorization was applied and the universally optimal design was presented when the population is homogeneous. In heterogeneous populations Pericleous and Kounias first applied the concept of mazorization and then found A-, D- and MV-optimal designs.

The definitions of information function and information matrix were given in (Pericleous and Kounias, 2011), in the line followed by Pukelsheim (1993). The definitions of information function and information matrix are given. Homogeneous populations set in a row with dependent observations are examined and properties of the optimal designs are presented, two treatments are studied in more detail and the optimal designs are given. Non homogeneous populations by one characteristic set in p rows and q columns, with independent observations, are analyzed and examples of Balanced Treatments Block designs are given.

Row designs are examined (Pericleous and Kounias 2012) with three treatments, homogeneous population with dependent observations. The dependence follows a first order autoregression $(\mathrm{AR}(1))$. A filtering procedure was presented to reduce the number of competing designs.

In the case of optimal designs in $3^{k}$ fractional factorial for the estimating linear and quadratic contrasts, most of the works for constructing optimal designs for parameter estimation in fractional factorials are concentrated in factors at two levels. It is of interested estimating linear and quadratic contrasts, in fractional factorials, with each factor at three levels. The books by Dey and Mukerjee (1999) and $\mathrm{Wu}$ and Hamada (2000), cover the topic, with a lot references. If the number of runs is $\mathrm{N}=0 \bmod 9$, the orthogonal arrays, $\mathrm{OA}(\mathrm{N} ; \mathrm{k} ; 3 ; 2)$, are $\Phi$-optimal under different type of criteria, $\operatorname{Kiefer}(1958,1960)$. If $\mathrm{N}=1 \bmod 9$ the plan obtained by augmentation of a run to an $\mathrm{OA}(\mathrm{N}-1 ; \mathrm{k} ; 3 ; 2)$ is D-optimal, KolyvaMachera (1989a), G-optimal, Kolyva-Machera(1989b), and optimal under Cheng's type 1 criteria, Mukerjee (1999). These efforts were concentrated in adding runs to an $\mathrm{OA}(\mathrm{N} ; \mathrm{k} ; 3 ; 2)$ so that the resulting design is optimal in some sense.

Chatzopoulos et al. (2009), studied the optimality of designs obtained by adding $p$ runs to an orthogonal array for experiments involving $m$ factors each at $s$ levels. The optimality criterion used was the Type 1 criterion due to Cheng (1978) which is an extension of Kiefer (1975) universal optimality criterion. Unlike what happens with orthogonal array plus one run designs, the behavior of designs obtained via augmentation of an orthogonal array by $p$ runs depends on the particular runs added. Onea et al. (2009) investigated two techniques of constructing optimal weighing designs under different optimality criteria when the number of runs is $N \equiv 3(\bmod 4)$. The first method was presented by Chatzopoulos, Kolyva - Machera and Chaterjee (2009), who studied Type I optimality criteria for $\mathrm{s}^{\mathrm{m}}$ factorial designs obtained by adding p particular runs to an orthogonal array of strength two. In the present work the orthogonal arrays $\mathrm{OA}(\mathrm{N}-3, \mathrm{~m}, 2,2)$ are augmented by three runs. The second one is due to Ehlich (1964). In order to compare the two methods they constructed the corresponding information matrices, applied D-, E- and A- optimality criteria and explored their efficiency. Chatterjee et al. (2011) considered the issue of optimality of fractional factorial experiments involving $\mathrm{m}$ factors each at two levels. The optimality criteria used here are the type 1 criteria, which include the D- and Acriteria. It is shown that if there exists an orthogonal array $\mathrm{OA}(\mathrm{N}-\ell ; \mathrm{m} ; 2 ; 3), \ell=1,2$, then there exists an n-run type 1 optimal fractional factorial plan for a $2^{\mathrm{m}}$ experiment under a model that includes the mean, all main effects and all two-factor interactions with a factor in common. These plans are obtained by adding any one run to an $\mathrm{OA}(\mathrm{N}-1 ; \mathrm{m} ; 2 ; 3)$ for $N \equiv 3(\bmod 8)$ and two specific runs to an $\mathrm{OA}(\mathrm{N}-2 ; \mathrm{m} ; 2 ; 3)$ for $N \equiv 2(\bmod 8)$.

In this project we will deal with factorial designs with factors, each at 3 levels, the primary interest is the estimation of linear and quadratic contrasts of factor's effects. Orthogonal designs, called orthogonal balanced arrays (OBA), are derived for any value of the number of experimental runs, in which the estimators of linear contrasts are uncorrelated with those of quadratic contrasts.

Another issue of interesting in experimental designs is saturated designs. An experimental design is said to be saturated if all degrees of freedom are consumed by the estimation of parameters, leaving no degrees of freedom for error variance estimation. Saturated resolution III factorial designs are commonly used in screening experiments, to determine which of many factors affects the measure of pertinent 
quality characteristics. The purpose of this paper is to give saturated resolution III designs, minimizing the generalized variance of the main effects and the general mean, that is, D-optimal designs. In recent years, there has been a considerable interest in optimal saturated main effect designs. Mukerjee et al. (1986) and Kraft (1990) showed that in the two-factor case all such designs are equivalent with respect to D-optimality. Later Mukerjee and Sinha (1990) considered optimality results on almost saturated main effect designs in the two-factor case. The result was also obtained by Pesotan and Raktoe (1988) in the special case of $2^{m}$ factorials, who also worked on a subclass of $3^{m}$ factorials. The first attempt to extend the two factor results to three factors was done by Chatterjee and Mukerjee (1993). Later Chatterjee and Narasimhan (2002) using techniques from Graph Theory and Combinatorics, claimed about the upper bound of the determinant of the saturated $3 \times m_{2} \times m_{3}$ factorials. Chatzopoulos and Machera (2002) considered the class of $m_{1} \times m_{2} \times m_{3}, m_{1} \geq 2, m_{2} \geq m_{1}, m_{3} \geq m_{2}$ factorial plans with the minimum number of observations. Their interest was to find conditions in order to maximize the determinant of the information matrix and compare their findings with other researchers' findings. Chatzopoulos and Kolyva-Machera (2005), considered the problem of finding an algorithm in order to construct D-optimal saturated factorial designs with three factors. The interest was to estimate the general mean and main effects while all higher order interactions are negligible, that is Resolution III plans. In order to reduce the steps of the algorithm, they diminish the number of the cases and have to search by studying the design matrix. The algorithm they suggested, leads in finding the upper bound of the determinant of the design and moreover in constructing the D-optimal design. They apply an algorithm in order to find the D-optimal saturated $4 \times 4 \times m_{3}, m_{3} \geq 6$ design and compare their results with those are mentioned in bibliography. Moreover they verify the findings for the $4 \times 4 \times 4$ and $4 \times 4$ $\times 5$ D-optimal saturated designs and expand their results by applying the algorithm for the saturated 4 $\times 5 \times 5$ and $4 \times 5 \times 6$ designs. Moreover, Chatzopoulos and Kolyva-Macher (2006) extended the results concerning D-optimal saturated main effect designs for $2 \times m_{2} \times m_{3}$ to $3 \times m_{2} \times m_{3}$ factorials, when $3 \leq m_{2} \geq 6$ and $m_{3} \geq m_{2}$. Recently Karagiannis and Moyssiadis (2005, 2008) extended the above cases and studied the D-optimal $3 \times m_{2} \times m_{2}$ and $3 \times m_{2} \times\left(m_{2}+1\right)$ saturated designs.

Although the issue has been studied by many researchers it is not exhausted. This area of research is far from complete because of the difficulty of the calculations for designs that are not symmetrical. Optimal designs are found only in certain cases.

\section{Majorization}

The information of interest is contained in the variance matrix, $\mathbf{V}=\sigma^{2} \mathbf{Q}^{-1}$, or equivalently in the information matrix $\mathbf{Q}$. There are several optimality criteria in bibliography. The most frequently used optimality criteria are:

G-optimality: If $E\left(y_{i}\right)=f^{\prime}\left(\mathbf{x}_{i}\right) \theta, f\left(\mathbf{x}_{i}\right)=\left(f_{1}\left(\mathbf{x}_{i}\right), \ldots, f_{p}\left(\mathbf{x}_{i}\right)\right)^{\prime}, i=1, \ldots, n$ is the linear model, then the variance response surface in points $\mathbf{x}_{i}, i=1, \ldots, n$, is $\operatorname{var}\left(f^{\prime}\left(x_{i}\right) \hat{\theta}\right)=\sigma^{2} f^{\prime}\left(x_{i}\right) \mathbf{V} f\left(x_{i}\right)$ and for Goptimality: $\min _{d} \max _{i} f^{\prime}\left(\mathbf{x}_{i}\right) \mathbf{V} f\left(\mathbf{x}_{i}\right)$.

G-optimality criterion is "a prediction criterion". This criterion latterly is called global or Goptimality. The aim of G-optimality is a response estimation criterion and can be defined as minimizing the maximum variance of any predicted value over the experimental space.

\section{D-optimality: $\max _{d} \operatorname{det}\left(\mathbf{Q}_{d}\right)$.}

D-optimality is a quite popular criterion maximizing the determinant of the information matrix, in essence D-optimality chooses that design as the "best" for which the volume (expected volume) of the joint confidence ellipsoid of the parameters of interest is minimized.

A-optimality: $\min _{d}\left(\right.$ trace $\mathbf{V}_{d}$ ) or $\max _{d}\left(\text { trace } \mathrm{Q}_{d}^{-1}\right)^{-1}$.

A-optimality is defined as minimizing the average variance of the parameter estimates, that is, minimizing the average variance of the best linear unbiased estimator (BLUE) of the parameters of interest.

E-optimality: $\min _{d} \max _{i} \lambda_{i}(\mathbf{V})$ or $\underbrace{\max }_{d} \underbrace{\min }_{i} \lambda_{i}(\mathbf{Q})$. 
E-optimality is defined as maximizing the minimum eigenvalue of the information matrix or equivalently, minimizing the maximum eigenvalue of the inverse of the information matrix. The aim of E-optimality is to minimize the maximum variance of all possible normalized linear combinations of parameter estimates.

$\boldsymbol{M V}$-optimality: $\min _{d} \max _{i}\left(v_{i i}\right), \quad \mathbf{V}=\left(v_{i j}\right)$.

MV-optimality is defined as minimizing the maximum variance of the parameters of interest.

Instead of dealing with specific optimality criteria is preferable to work with universal optimality, an idea introduced by Kiefer (1975), and $\Phi$-optimality and if such designs do not exist, then go to the specific criteria mentioned above.

Universal and $\Phi$-optimality are defined below:

Definition 1: If $C \in \operatorname{nnd}(k)$ the function $\varphi(\mathbf{C})$ is called information function if $\varphi(\mathbf{C}) \rightarrow \mathbb{R}$, and the following conditions are satisfied:

(i) $\mathbf{C} \geq \mathbf{D} \Rightarrow \varphi(\mathbf{C}) \geq \varphi(\mathbf{D})$

(ii) $\varphi(p \mathbf{C}+(1-p) \mathbf{D}) \geq p \varphi(\mathbf{C})+(1-p) \varphi(\mathbf{D}) \quad \forall 0<p<1, \mathbf{C}, \mathbf{D} \geq \mathbf{0}$

(iii) $\varphi(d C)=d \varphi(C), \quad \forall d>0, \mathbf{C} \geq \mathbf{0} \Rightarrow \varphi(\mathbf{0})=0 \Rightarrow \varphi(\mathbf{C}) \geq 0, \forall \mathbf{C} \geq \mathbf{0}$

(iv) $\varphi(\mathbf{C})=\varphi\left(\mathbf{P C P}^{\prime}\right) \quad \forall \mathbf{C} \in \operatorname{nnd}(k), \mathbf{P} \in \operatorname{perm}(k)$

So the information functions are concave and increasing. Here $n n d(k)$ is for non negative definite $k \times k$ matrix, $\operatorname{perm}(k)$ is for permutation $k \times k$ matrix.

Definition 2: A design $d^{*}$ with information matrix $\mathbf{C}_{d}^{*}$ is universally optimal, in the class $\mathrm{F}$ of designs, if it maximizes $\varphi\left(\mathbf{C}_{d}\right), d \in F$, for all information functions $\varphi$.

Definition 3: A design $d^{*}$ with information matrix $\mathbf{C}_{d}^{*}$ is $\Phi$-optimal, in the class $\mathrm{F}$ of designs, if $\sum_{i=1}^{v} \varphi\left(\lambda_{i}\right) \leq \sum_{i=1}^{v} \varphi\left(\mu_{i}\right)$ for all continuous, decreasing convex functions $\varphi$, where $\lambda=\left(\lambda_{1}, \ldots, \lambda_{v}\right)$ are the latent roots of $\mathbf{C}_{d}^{*}$ and $\mu=\left(\mu_{1}, \ldots, \mu_{v}\right)$ are the latent roots of $C_{d} \forall d \in F$.

This is equivalent to: $\lambda \prec \mu$, that is, the vector $\lambda$ is majorized by the vector $\mu$ (Marshall and Olkin p.10).

If a design is universally optimal it is also $\Phi$-optimal and if a design is $\Phi$-optimal, it is A-, D-, Eoptimal. Also a design may be optimal for a criterion but not optimal for the other criteria. Note that MV-optimality is not covered by $\Phi$-optimality, since it cannot be expressed as a function of the latent roots of the information matrix.

The idea is to use the concept of majorization in order to establish $\Phi$ optimality.

\section{$3 \quad$ Fields of Applications}

They will be present in three fields of application of the concept of majorization, one for each different case of experimental designs.

1) The first idea is for Repeated measurements of p periods, two treatments, $2^{p}$ sequences (we use the symbolism $s_{i} i=0,1, \ldots, m$, where $\left.m=2^{p}-1\right)$.

For the estimation of the parameters we have:

If $\mathrm{n}$ is the total number of experimental units and $u_{i} i=0,1, \ldots, m$ is the number of experimental units that the sequence $s_{i}$ of treatments is applied, then $u_{0}+u_{1}+\ldots+u_{m}=n$. The model is:

$$
Y_{i j}=\tau_{d(i, j)}+\pi_{j}+\delta_{d(i, j-1)}+\gamma_{i}+e_{i j}
$$

where, $i=1, \ldots, n$, refers to the unit employed, $j=1, \ldots, p$ the period, $\tau_{d(i j)} \in\left\{\tau_{\mathrm{A}}, \tau_{\mathrm{B}}\right\}$ is the direct effect of the treatment applied, under design $\mathrm{d}$, in the $j$ th period on the ith unit, $\delta_{d(i, j-1)} \in\left\{\delta_{\mathrm{A}}, \delta_{\mathrm{B}}\right\}$ is the residual effect of the treatment applied the $(j-1)$ th period on the $i$ th unit, $\pi_{j}$ is the $j$ th period effect and $\gamma_{i}$ is the $i$ th unit effect. The errors $e_{i j}$ are independent within each unit and among units and have 0 mean and constant variance. 
The model (1) in vector form is written:

$$
\mathbf{Y}=\tau_{\mathrm{A}} \tau_{\mathrm{A}}+\tau_{\mathrm{B}} \tau_{\mathrm{B}}+\delta_{\mathrm{A}} \delta_{\mathrm{A}}+\delta_{\mathrm{B}} \delta_{\mathrm{B}}+\pi_{1} \pi_{1}+\cdots+\pi_{p} \pi_{p}+\gamma_{1} \gamma_{1}+\cdots \gamma_{n} \gamma_{n}+e
$$

where $\mathrm{Y}, \tau_{\mathrm{A}}, \tau_{\mathrm{B}}, \delta_{\mathrm{A}}, \delta_{\mathrm{B}}, \pi_{1}, \ldots, \pi_{p}, \gamma_{1}, \ldots, \gamma_{n}, e$ are $p n$ vectors, and $\tau_{\mathrm{A}}\left(\tau_{\mathrm{B}}\right)$ has 1 when treatment $\mathrm{A}(\mathrm{B})$ is applied and 0 elsewhere, that is $\tau_{\mathrm{A}}+\tau_{\mathrm{B}}=1_{p n}, \delta_{\mathrm{A}}\left(\delta_{\mathrm{B}}\right)$ has 0 in the first period, 1 when treatment $\mathrm{A}(\mathrm{B})$ was applied in the previous period and 0 elsewhere, $\pi_{i}$ has 1 in the ith period and 0 elsewhere, thus $\delta_{\mathrm{A}}+\delta_{\mathrm{B}}+\pi_{1}=1_{p n}, \pi_{1}+\cdots \pi_{p}=1_{p n}$. Also $\gamma_{i}, i=1, \ldots, n$ has 1 when the ith unit is employed and 0 e1sewhere, that is, $\gamma_{1}+\cdots+\gamma_{n}=1_{p n}$.

For the estimation of the parameters of interest not all the other parameters are used (Kounias and Chalikias 2008b). By using the idea of majorization in least squares method, Chalikias and Kounias (2012) have given necessary conditions for $\Phi$-optimality for estimating treatment and residual effects and improved conditions given by Cheng and $\mathrm{Wu}$ (1980). This work can be extended to three and more treatments.

2) Another idea is at the Optimal Designs with three treatments in row-column and in factorial designs

The experimental units are arranged in row, there are $\mathrm{v}$ treatments and to every unit one of the $\mathrm{v}$ treatments is applied. The population is homogeneous with dependent observations. The model in vector form is $y=\mu_{1} x_{1}+\cdots+\mu_{v} x_{v}+e, E\left(e e^{\prime}\right)=\sigma^{2} v$, where the $n \times 1$ vector $x_{j}=\left(x_{1 j}, \ldots, x_{n j}\right)^{\prime}$ has $x_{i j}=1$ when the jth treatment is applied to the ith unit and 0 elsewhere. The errors follow a first order autoregressive $\operatorname{AR}(1)$ scheme: $e_{i}-a e_{i-1}=w_{i}, i=1,2, \ldots, n,|a|<1$, and $w_{i}$ are uncorrelated random variables with $E\left(w_{i}\right)=0, E\left(w_{i}^{2}\right)=\sigma^{2}$. So we have $\operatorname{cov}\left(e_{i}, e_{j}\right)=\sigma^{2} a^{|i-j|} /\left(1-a^{2}\right)$ and the inverse of the $n \times n$ covariance matrix has the form:

$$
\mathbf{V}^{-1}=\mathbf{I}_{\mathbf{n}}+a^{2}\left[\begin{array}{ccc}
0 & \cdots & 0 \\
\vdots & \mathbf{I}_{\mathbf{n}-2} & \vdots \\
0 & \cdots & 0
\end{array}\right]-a\left[\begin{array}{cccc}
0 & 1 & 0 & 0 \\
1 & \ddots & \ddots & 0 \\
0 & \ddots & \ddots & 1 \\
0 & 0 & 1 & 0
\end{array}\right]
$$

The parameters of interest are $\mu_{1}, \ldots, \mu_{v}$. The information matrix $\mathbf{Q}$ is: $\mathbf{Q}=\mathbf{X}^{\prime} \mathbf{V}^{-1} \mathbf{X}, \quad \mathbf{X}=\left(\mathbf{x}_{1}, \ldots, \mathbf{x}_{v}\right)$, so

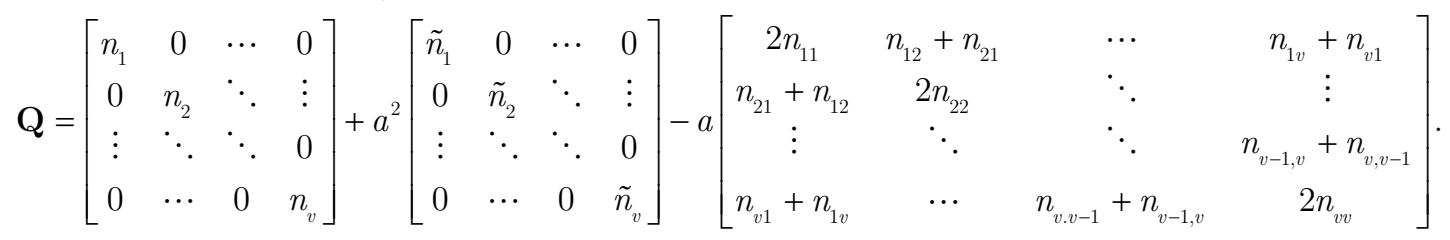

where,

$n_{i} \quad i=1, \ldots, v$ is the number of times treatment $T_{i}$ is applied to the $\mathrm{n}$ units $1,2, \ldots, \mathrm{n}$.

$\tilde{n}_{i} i=1, \ldots, v$ is the number of times treatment $T_{i}$ appears in the $\mathrm{n}-2$ inside units $2,3, \ldots, \mathrm{n}-1$.

$n_{i i} \quad i=1, \ldots, v$ is the number of times the pair $T_{i} T_{i}$ appears in the positions $12,23, \ldots,(\mathrm{n}-1) \mathrm{n}$.

$n_{i j} \quad i \neq j \quad i, j=1, \ldots, v$ is the number of times the pair $T_{i} T_{j}$ appears in the positions $12,23, \ldots,(\mathrm{n}-1) \mathrm{n}$.

Then applying the averaging rule (Kiefer, 1975) and using the concept of majorization can be found some general rules for the case where the value of a is negative and the case where the value of a is positive. It seems that when a is negative it is easier to handle.

It is proved that: (i) When $0<a<1$ in the universally optimal design at most one treatment has a run with length $n_{i i}>0$. (ii) If $-1<a<0$ in the optimal design every pair of distinct treatments appear at most once.

In the case of two treatments $\mathrm{S}, \mathrm{T}$ and $\mathrm{n}$ even: (i) If $0<a<1$ the universally optimal design is STST...ST (ii) If $-1<a<0$ the universally optimal design is SS..STT...T with $\mathrm{S}$ and $\mathrm{T}$ in equal numbers. 
The model (1) in vector form is written:

$$
\mathbf{Y}=\tau_{\mathrm{A}} \tau_{\mathrm{A}}+\tau_{\mathrm{B}} \tau_{\mathrm{B}}+\delta_{\mathrm{A}} \delta_{\mathrm{A}}+\delta_{\mathrm{B}} \delta_{\mathrm{B}}+\pi_{1} \pi_{1}+\cdots+\pi_{p} \pi_{p}+\gamma_{1} \gamma_{1}+\cdots \gamma_{n} \gamma_{n}+e
$$

where $\mathrm{Y}, \tau_{\mathrm{A}}, \tau_{\mathrm{B}}, \delta_{\mathrm{A}}, \delta_{\mathrm{B}}, \pi_{1}, \ldots, \pi_{p}, \gamma_{1}, \ldots, \gamma_{n}, e$ are $p n$ vectors, and $\tau_{\mathrm{A}}\left(\tau_{\mathrm{B}}\right)$ has 1 when treatment $\mathrm{A}(\mathrm{B})$ is applied and 0 elsewhere, that is $\tau_{\mathrm{A}}+\tau_{\mathrm{B}}=1_{p n}, \delta_{\mathrm{A}}\left(\delta_{\mathrm{B}}\right)$ has 0 in the first period, 1 when treatment $\mathrm{A}(\mathrm{B})$ was applied in the previous period and 0 elsewhere, $\pi_{i}$ has 1 in the ith period and 0 elsewhere, thus $\delta_{\mathrm{A}}+\delta_{\mathrm{B}}+\pi_{1}=1_{p n}, \pi_{1}+\cdots \pi_{p}=1_{p n}$. Also $\gamma_{i}, i=1, \ldots, n$ has 1 when the ith unit is employed and 0 e1sewhere, that is, $\gamma_{1}+\cdots+\gamma_{n}=1_{p n}$.

For the estimation of the parameters of interest not all the other parameters are used (Kounias and Chalikias 2008b). By using the idea of majorization in least squares method, Chalikias and Kounias (2012) have given necessary conditions for $\Phi$-optimality for estimating treatment and residual effects and improved conditions given by Cheng and $\mathrm{Wu}$ (1980). This work can be extended to three and more treatments.

2) Another idea is at the Optimal Designs with three treatments in row-column and in factorial designs

The experimental units are arranged in row, there are $\mathrm{v}$ treatments and to every unit one of the $\mathrm{v}$ treatments is applied. The population is homogeneous with dependent observations. The model in vector form is $y=\mu_{1} x_{1}+\cdots+\mu_{v} x_{v}+e, E\left(e e^{\prime}\right)=\sigma^{2} v$, where the $n \times 1$ vector $x_{j}=\left(x_{1 j}, \ldots, x_{n j}\right)^{\prime}$ has $x_{i j}=1$ when the jth treatment is applied to the ith unit and 0 elsewhere. The errors follow a first order autoregressive $\operatorname{AR}(1)$ scheme: $e_{i}-a e_{i-1}=w_{i}, i=1,2, \ldots, n,|a|<1$, and $w_{i}$ are uncorrelated random variables with $E\left(w_{i}\right)=0, E\left(w_{i}^{2}\right)=\sigma^{2}$. So we have $\operatorname{cov}\left(e_{i}, e_{j}\right)=\sigma^{2} a^{|i-j|} /\left(1-a^{2}\right)$ and the inverse of the $n \times n$ covariance matrix has the form:

$$
\mathbf{V}^{-1}=\mathbf{I}_{\mathbf{n}}+a^{2}\left[\begin{array}{ccc}
0 & \cdots & 0 \\
\vdots & \mathbf{I}_{\mathbf{n}-2} & \vdots \\
0 & \cdots & 0
\end{array}\right]-a\left[\begin{array}{cccc}
0 & 1 & 0 & 0 \\
1 & \ddots & \ddots & 0 \\
0 & \ddots & \ddots & 1 \\
0 & 0 & 1 & 0
\end{array}\right]
$$

The parameters of interest are $\mu_{1}, \ldots, \mu_{v}$. The information matrix $\mathbf{Q}$ is: $\mathbf{Q}=\mathbf{X}^{\prime} \mathbf{V}^{-1} \mathbf{X}, \quad \mathbf{X}=\left(\mathbf{x}_{1}, \ldots, \mathbf{x}_{v}\right)$, so

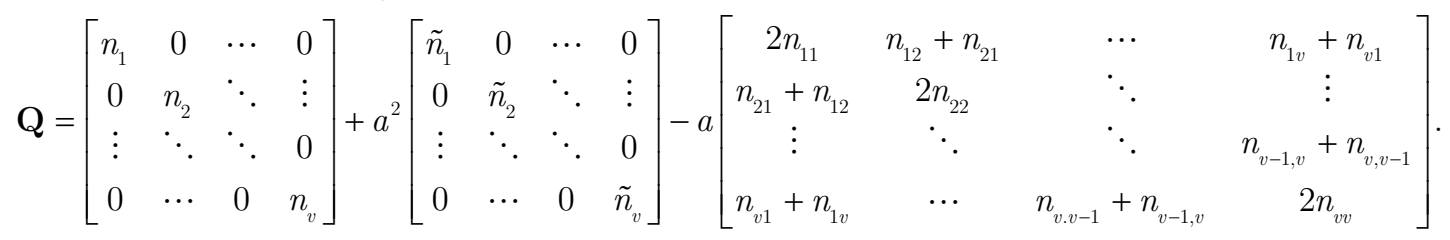

where,

$n_{i} \quad i=1, \ldots, v$ is the number of times treatment $T_{i}$ is applied to the $\mathrm{n}$ units $1,2, \ldots, \mathrm{n}$.

$\tilde{n}_{i} i=1, \ldots, v$ is the number of times treatment $T_{i}$ appears in the $\mathrm{n}-2$ inside units $2,3, \ldots, \mathrm{n}-1$.

$n_{i i} \quad i=1, \ldots, v$ is the number of times the pair $T_{i} T_{i}$ appears in the positions $12,23, \ldots,(\mathrm{n}-1) \mathrm{n}$.

$n_{i j} \quad i \neq j \quad i, j=1, \ldots, v$ is the number of times the pair $T_{i} T_{j}$ appears in the positions $12,23, \ldots,(\mathrm{n}-1) \mathrm{n}$.

Then applying the averaging rule (Kiefer, 1975) and using the concept of majorization can be found some general rules for the case where the value of a is negative and the case where the value of a is positive. It seems that when a is negative it is easier to handle.

It is proved that: (i) When $0<a<1$ in the universally optimal design at most one treatment has a run with length $n_{i i}>0$. (ii) If $-1<a<0$ in the optimal design every pair of distinct treatments appear at most once.

In the case of two treatments $\mathrm{S}, \mathrm{T}$ and $\mathrm{n}$ even: (i) If $0<a<1$ the universally optimal design is STST...ST (ii) If $-1<a<0$ the universally optimal design is SS..STT...T with $\mathrm{S}$ and $\mathrm{T}$ in equal numbers. 
For $n$ odd and two treatments the results are not so simple (i) $0<a<1$ the competing designs for optimality are SSTSTS...TST and STST...STS. (ii) $-1<a<0$ the competing designs for optimality are SS...STT...T and SS...STT...TS where the numbers of the two treatments differ by 1.

The case of three treatments is more complicated and applying majorization optimal designs under dependence can be found. Also the study can be extended to non homogeneous populations by one or two characteristics, when the number of treatments is three.

3) Another problem is to find optimal designs for $3^{k}$ fractional factorial designs for estimating linear and quadratic contrasts.

The problem of finding optimal designs under different types of criteria preoccupied many researchers in the last decades. Most of them dealt with $2^{m}$ fractional factorial designs and some cases of general asymmetrical $m_{1} \times m_{2} \times \ldots \times m_{k}$ factorials.

Using majorization, as applied to optimal designs Pukelsheim (1993 p.139-142, 352-358) another approach can be used and optimal designs, for estimating linear and quadratic contrasts, for any value of $N$. For this the concept of Balanced Arrays $\mathrm{B}(N, k)$ and Orthogonal Balanced Arrays $\operatorname{OBA}(N, k)$, is introduced, that is, $N \times k$ arrays with three symbols $0,1,2$, where $N$ is the number of runs and $k$ is the number of factors involved. The parameters of the $\operatorname{OBA}(N, k)$ will be specified and optimal designs will be given for all values $N$.

Moreover, a demanding problem is to find the maximum number $k$ of factors that we can accommodate. An upper bound for the maximum number of factors is $(N-1) / 2$. This bound is not attained in most of the cases.

\section{Conclusions}

The concept of majorization can be a useful tool for designing experiment. Majorization concept can be used to prove $\Phi$-optimality and usually is the only way to do that. As we have seen there are many fields in which majorization can be used and the usage of the method in more fields of experimental design is of interest.

\section{References}

1. Agrawal, H. (1966). Some generalizations of distinct representative with applications to statistical designs. Ann. Math. Statist. 37, 525-528.

2. Bechhofer, R.E. and Tamhane, A.C. (1981). Incomplete block designs for comparing treatments with a control: General Theory. Technometrics, 23, 45-57.

3. Chalikias, M. S. (2017). Two Treatment Repeated Measurement Designs With Uncorrelated Observations: A Compact Review. Journal of Advanced Statistics, 2(1), 27.

4. Chalikias, M., Kounias S., 2012, "Extension and necessity of Cheng and Wu conditions" Journal of Statistical Planning and Inference 142 (7), pp. 1794-1800.

5. Chalikias M., Kounias S., (2017) Optimal two Treatment Repeated Measurement Designs for three Periods Communications in Statistics - Theory and Methods Vol 46, Pages: 200-209

6. Chatzopoulos, St. A., and Kolyva-Machera, F. (2006). Some D-Optimal Saturated Designs for $3 \times \mathrm{m}_{2} \times \mathrm{m}_{3}$ Factorials". J. Statist. Plann. Inference, 136, 2820-2830.

7. Chatzopoulos, St. A., Kolyva-Machera, F. (2005). Algorithmic Finding of D-Optimal Saturated $\mathrm{m}_{1} \mathrm{xm}_{2} \mathrm{xm}_{3}$ Designs. Proceedings of $18^{\text {th }}$ Annual Conference of Greek Statistical Institute, 355-362.

8. Chatzopoulos, St. A., and Kolyva-Machera, F. (2002). D-Optimal $\mathrm{m}_{1} \times \mathrm{m}_{2} \times \mathrm{m}_{3}$ saturated factorial designs. Proceedings of $15^{\text {th }}$ Annual Conference of Greek Statistical Institute, 664-671.

9. Chatzopoulos, St. A., Kolyva-Machera, F., Chatterjee, K. (2009). Optimality Results on Orthogonal Arrays Plus p Runs for s ${ }^{\mathrm{m}}$ Factorial Experiment. Metrika, 73: 385-394.

10. Chatterjee, K., Narasimhan, G. (2002). Graph theoretic techniques in D-optimal design problems. J. Statist. Plann. Inference 102, 377-387.

11. Chatterjee, K., Mukerjee, R. (1993). D-optimal saturated main effect plans for $2 \times s_{2} \times s_{3}$ factorials. J. Combin. Inform. System Sci. 18, 116-122. 
12. Chatterjee, K., Kolyva-Machera, F., Chatzopoulos, St. A. (2011). Type 1 Optimal $2^{\mathrm{m}}$ Fractional Factorial Plans with $n \equiv \ell(\bmod 8)$ Runs, $\ell=1,2$. Journal of the Korean Statistical Society, 40, 451-455.

13. Chauhan, N. (2000). Efficient and optimal designs for correlated errors. Ph.D. Dissertation, University of Sheffield.

14. Chauhan, N. And Martin, R.J. (2001). Optimal nested row-collumn designs for blocks of size 2x2 under dependence. Metrika 53, 1-14.

15. Cochran W.G. and Cox G.M. (1957). Experimental Design. John Wiley and Sons, Inc.

16. Das, A. and Dey, A. (1990). Universal optimality and non-optimality of some row-column designs. J. Statist. Plann. Inference 31, 263-271.

17. Dey, A. and Mukerjee, R. (1999). Fractional Factorial Plans. New York: Wiley.

18. Ehlich, H. (1964). Determinantenabschatzungen fur binare matrizen mit $n=3 \bmod 4$. Math. Zeitschr. 84, 438-447.

19. Harley, H. O. and Smith, C. A. B. (1948). The construction of Youden squares. J. Roy. Statist. Soc., SER. B 10, 262-263.

20. Hedayat, A.S., Jacroux, M., Majundar, D. (1988). Optimal designs for comparing test treatment with controls. Statistical Science Vol.3, No. 4, 462-491.

21. Hedayat, A.S., Majundar, D. (1985). Combining experiments under Gauss-Markov models. Journal of the American Statistical Association 80,698-703.

22. Hedayat, A. S., Sloane, N. J. A., Stufken, J. (1999). Orthogonal Arrays: Theory and Applications: Springer Series in Statistics.

23. Jacroux, M. (1987). Some E and MV-optimal row-column designs having equal numbers of rows and columns. Metrika 6, 361-381.

24. Karagiannis, V. and Moyssiadis, C. (2005). Construction of D-optimal s1Xs2 Xs3 factorial designs using graph theory. Metrika 62, 283-307.

25. Karagiannis, V. and Moyssiadis, C. (2008). Construction A graphical construction of the D-optimal saturated, $3 \mathrm{xs}^{2}$ main effect, factorial design. J. Statist. Plann. Inference 138, 1679-1696.

26. Kiefer, J. (1958). On the nonrandomized optimality and randomized non-optimality of symmetrical designs. Ann. Math. Statist. 29, 675-699.

27. Kiefer, J. (1975 a). Construction and optimality of generalized Youden designs. In: J.N. Srivastava, ed., A Survey of Statistical Designs and Linear Models. North-Holland, Amsterdam, 333-353.

28. Kiefer, J. (1975 b). Balanced block designs and generalized Youden designs, I. Construction (Patchwork). The Annals of StatisticsVol.3, No.1, 109-118.

29. Kolyva-Machera, F. (1989a). D-optimality in 3k designs for $N-1 \bmod 9$ observations. J. Statist. Plann. Inference 22, 95-103.

30. Kolyva-Machera, F. (1989b). Fractional factorial designs and G-optimality. In Proceedings Forth Prague Symp. on Asymptotic Statistics, 349-358, Prague, Charles University Press.

31. Kounias, S. (2008). Optimal designs in heterogeneous populations, Proceedings of $20^{\text {th }}$ Annual Conference of Greek Statistical Institute, 209-216.

32. Kounias S. (2009). Optimal designs in populations with two treatments and dependent observations. Proceedings of $21^{\text {th }}$ Annual Conference of Greek Statistical Institute, 195-202.

33. Kounias, S., Chalikias, M., 2008a, "Optimal and universally optimal two treatment repeated measurement designs", in Vonta, F. et al., "Statistics for industry and technology", edit. Birkhauser, Boston, Basel, Berlin 2008, pp. $465-477$.

34. Kounias, S., Chalikias M., 2008b, "Estimability of parameters in a linear model and related characterisations", Statistics and Probability Letters 78 (15), pp. 2437-2439.

35. Kounias, S., \& Chalikias M.S.,(2015) "Optimal two treatment repeated measurement designs with treatmentperiod interaction in the model" UTILITAS MATHEMATICA, 96, 243-261.

36. Kounias S, Pericleous K (2011). Optimal row designs with three treatments under dependence, (It is posted on the conference website).

37. Kounias S, Pericleous S (2012). The concept of majorization in experimental design, Communications in Statistics-Theory and Methods, (accepted for publication).

38. Kraft, O. (1990). Some matrix representations occurring in two factor models. In Probability, Statistics and Design of Experiments (R.R. Bahadur, ed.), pp. 461-470, New Delhi: Wiley Eastern. 
39. Marshal, A.A. and Olkin, I. (1979). Inequalities: Theory of majorization and its applications. Academic Press, New York.

40. Martin, R.J. and Eccleston, J.A. (1993). Incomplete block designs with spatial layout when observations and dependent. J. Statist. Plann. Inference 35, 77-92.

41. Morgan, J.P. and Uddin, N. (1991). Two-dimensional designs for correlated errors. Ann. Statist. 19, $2160-2182$.

42. Morgan, J.P. and Uddin, N. (2003). Optimal row-column designs for two treatments. J. Statist. Plann. Inference $115,603-622$.

43. Mukerjee, R., Chatterjee, K., Sen, M. (1986). D-optimality of a class of saturated main effect plans and allied results. Statistics 17 (3), 349-355.

44. Mukerjee, R., Sinha, B.K. (1990). Almost saturated D-optimal main effect plans and allied results. Metrika 37, 301-307.

45. Mukerjee, R. (1999). On the optimality of orthogonal array plus one run plans. Ann. Statist 27, 1256-1271.

46. Onea, M. A., Kolyva - Machera, F., Chatzopoulos, St. A. (2010). Type 1 optimality of weighing designs with $N \equiv 3 \bmod 4$ runs. Proceedings of $23^{\text {th }}$ Annual Conference of Greek Statistical Institute, 430-437.

47. Pericleous K., Kounias S. (2010). The Concept of Mazorization in Experimental Designs. Proceedings of $22^{\text {th }}$ Annual Conference of Greek Statistical Institute, 167-174.

48. Pericleous K. (2011). Row-Column designs, optimization, two treatments, dependent observation. Proceedings of $23^{\text {th }}$ Annual Conference of Greek Statistical Institute, 239-246.

49. Pericleous K, Kounias S. (2012). Row-Column designs with three treatments and dependent observations. Proceedings of $24^{\text {th }}$ Annual Conference of Greek Statistical Institute, 280-287.

50. Pesotan, H., Raktoe, B.L. (1985). On invariance and randomization in factorial designs with applications to Doptimal main effect designs of the symmetrical factorial. J. Statist. Plann. Inference 19, 283-298.

51. Pukelsheim, F. (1993). Optimal designs of Experiments. John Wiley and Sons, Inc.

52. Raghavarao, D. (1971). Constructions and Combinatorial Problems in Design of Experiments. New York: Wiley.

53. Shrikhande, S.S.(1951). Designs with two-way elimination of heterogeneity.

54. Stufken, J. (1987). A-optimal block designs for comparing test treatments with a control. Annals of Statistics 15, 1629-1638.

55. Uddin, N. (1997). Row-column designs for comparing two treatments in the presence of correlated errors. J. Statist. Res. 31, 75-81.

56. Uddin, N. and Morgan, J.P. (1991). Optimal and near optimal sets of Latin squares for correlated errors. J. Statist. Plann. Inf. 29, 279-290.

57. Wald, A. (1943). On the efficient design of statistical investigations. Ann. Math. Statist. 14, 134-140. Ann. Math. Statist. 22, 245-247.

58. Wu, C.F.J. and Hamada, M. (2000). Experiments: planning, analysis, and parameter design optimization, Wiley, New York. 